\title{
Der Wegfall der Personenfreizügigkeit aufgrund des Brexits - Bestandesaufnahme der Beziehung
} Schweiz-UK

Am 1.Januar 2021 fiel zwischen der Schweiz und dem Vereinigten Königreich durch den Brexit das Freizügigkeitsabkommen (FZA) weg. Mit dem 2019 abgeschlossenen Abkommen über die erworbenen Rechte der Bürgerinnen und Bürger sollen die nach Art.23 FZA erworbenen Ansprüche geregelt und ausgestaltet werden. Grossteils bewahrt das neue Abkommen im Bereich der Freizügigkeit die Regelungen aus dem FZA, in gewissen Aspekten gestaltet es sich aber auch restriktiver, wobei sich die Frage nach dem Verhältnis zu Art.23 stellt. Für Personen, die keine erworbenen Ansprüche haben, kommt ein vollständig neues Regime zur Anwendung. So gibt es zwar ein befristetes Abkommen für die Dienstleistungserbringung. Im Übrigen wurde jedoch kein umfassendes Migrationsabkommen abgeschlossen und es kommen die nationalen Regelungen zur Anwendung. Insgesamt sind die Möglichkeiten zur Migration zwischen den beiden Ländern sehr viel begrenzter geworden.

I. Einleitung

II. Abkommen über die erworbenen Rechte der Bürgerinnen und Bürger

1. Grundzüge

2. Aufenthaltsrechte

3. Grenzgängerinnen und Grenzgänger

4. Dienstleistungserbringerinnen und -erbringer

5. Verhältnis zu Art.23 FZA

Zitiervorschlag:

SOFIE STELLER, Der Wegfall der Personenfreizügigkeit aufgrund des Brexits - Bestandesaufnahme der Beziehung Schweiz-UK, sui generis 2021, S. 209

Sofie Steller, MLaw, Diplomassistentin am Lehrstuhl für Europarecht und europäisches Migrationsrecht der Universität Fribourg und Assistentin im Völkerrecht an der FernUni Schweiz (sofie.steller@ unifr.ch). Die Autorin dankt Frau Prof. Sarah Progin-Theuerkauf für die hilfreiche Unterstützung.

URL: sui-generis.ch/184

DOI: https://doi.org/10.21257/sg.184

Dieses Werk ist lizenziert unter einer Creative Commons Namensnennung - Weitergabe unter gleichen Bedingungen 4.0 International Lizenz. 


\section{Einleitung}

1 Seit bald fünfJahren beschäftigt der Brexit, der Austrittsprozess des Vereinigten Königreichs (UK) aus der Europäischen Union (EU), die Öffentlichkeit. Die Schweiz blieb davon nicht unberührt. Dadurch, dass der Grossteil der Beziehungen zwischen der Schweiz und dem UK durch Abkommen zwischen der Schweiz und der EU bzw. ihren Mitgliedstaaten geregelt war ${ }^{1}$, mussten diese Beziehungen innerhalb vergleichsweise kurzer Frist neu geregelt werden. Besonders im Bereich der Migration, der in erster Linie durch das Freizügigkeitsabkommen (FZA) ${ }^{2}$ geregelt war, wurde schnell klar, dass der Brexit zu einem Bruch führen würde. Es war ausserdem klar, dass viele Personen davon betroffen sein würden. So lebten Ende 2019 rund 36'800 Schweizerinnen und Schweizer im UK; umgekehrt lebten rund 43'800 britische Staatsangehörige in der Schweiz. ${ }^{3}$ Hinzu kamen Ende 2018 insgesamt 433 Grenzgängerbewilligungen und 6'236 Meldungen für befristete Erwerbstätigkeit im Rahmen einer grenzüberschreitenden Dienstleistungserbringung bzw. einem Stellenantritt bei einem Unternehmen in der Schweiz. ${ }^{4}$

2 Die vorliegende kurze Untersuchung soll aufzeigen, wie der Austritt des UK aus der EU die Beziehungen zwischen der Schweiz und dem UK im Bereich Migration beeinflusst hat und wo diese einige Monate nach dem Ende der Übergangsphase stehen. Der Hauptteil dieses Beitrags widmet sich hierbei dem Abkommen über die Rechte der Bürgerinnen und Bürger, welches die unter dem FZA erworbenen Rechte in Zukunft schützen soll. Dabei wird kurz auf die Grundzüge dieses Abkommens eingegangen, um danach die Ausgestaltung der Aufenthaltsrechte sowie die Situation der Grenzgängerinnen und Grenzgänger sowie der Dienstleistungserbringer zu diskutieren. Hierbei spielt auch die Frage des Verhältnisses des neuen Abkommens zum FZA und speziell dessen Art.23 eine Rolle, besonders dort, wo das neue Abkommen vom FZA abweicht. Abschliessend soll ein kurzer Überblick über die neue Beziehung der Schweiz und des UK nach dem Wegfall der Personenfreizügigkeit gegeben werden. Insbesondere das neue Abkommen im Bereich der Dienstleistungserbringung soll dabei zur Sprache kommen.

1 Botschaft vom 6. Dezember 2019 zur Genehmigung und Umsetzung des Abkommens zwischen der Schweizerischen Eidgenossenschaft und dem Vereinigten Königreich von Grossbritannien und Nordirland über die Rechte der Bürgerinnen und Bürger infolge des Austritts des Vereinigten Königreichs aus der Europäischen Union und des Wegfalls des Freizügigkeitsabkommens (BBl 20201029), S. 1034.

2 Abkommen zwischen der Schweizerischen Eidgenossenschaft einerseits und der Europäischen Gemeinschaft und ihrer Mitgliedstaaten andererseits über die Freizügigkeit (FZA; SR 0.142.112.681).

3 CORNELIA LÜTHY, Arbeitsmarkt zwischen Schweiz und UK bleibt offen, Die Volkswirtschaft 4/2020, S.17.

4 Botschaft zum Abkommen (Fn.1), S. 1040.

\section{Abkommen über} die erworbenen Rechte der Bürgerinnen und Bürger

Im Nachgang des Brexit-Referendums entwickelte der 3 Bundesrat rasch seine «Mind the Gap»-Strategie - rechtliche Lücken beim Austritt des UK aus der EU sollten verhindert werden. ${ }^{5}$ Es wurde in verschiedenen Bereichen ein Handlungsbedarf identifiziert, insbesondere in jenem der «Bürgerrechte», welcher Gegenstand dieses Beitrags bilden soll. In erster Linie ging es dabei darum, die rechtliche Situation von Schweizer Staatsangehörigen im UK sowie von britischen Staatsangehörigen in der Schweiz nach dem Austritt zu klären. Im Zentrum stand die Sicherung der Rechte, die gestützt auf das Freizügigkeitsabkommen ${ }^{6}$ erworben worden sind, das ausschliesslich zwischen der EU und ihren Mitgliedstaaten einerseits und der Schweiz andererseits gilt und somit mit dem Ende der EU-Mitgliedschaft des UK auf dieses nicht mehr anwendbar ist. ${ }^{7}$ Zu diesem Zweck unterzeichneten die Schweiz und das UK am 25. Februar 2019 das Abkommen über die Rechte der Bürgerinnen und Bürger (im Folgenden: das Abkommen) ${ }^{8}$. Dieses wurde nach dem Wegfall des FZA zwischen der Schweiz und dem UK ab dem 1.Januar 2021 zunächst vorläufig angewendet, wodurch ein vertragsloser Zustand verhindert werden konnte. Am 1. März 2021 trat es durch Notenaustausch in Kraft. ${ }^{9}$

\section{Grundzüge}

Das Abkommen setzt sich, angelehnt an die Struktur des 4 FZA, aus fünf Teilen zusammen. Der vorliegende Beitrag

5 Botschaft zum Abkommen (Fn.1), S. 1030.

6 Eine grundlegende Behandlung des FZA würde den Rahmen dieses Beitrags übersteigen, siehe hierfür statt vieler: ASTRID EPINEY, Das Freizügigkeitsabkommen Schweiz-EU, in: Wollenschläger (Hrsg.), Europäischer Freizügigkeitsraum - Unionsbürgerschaft und Migrationsrecht, 2. Aufl., Zürich 2021, mit weiteren Verweisen; ALVARO BORGHI, La libre circulation des personnes entre la Suisse et l'UE, Commentaire article par article de l'accord du 21 juin 1999, Genf 2010; MARC SPESCHA, Migrationsrecht: Kommentar: Ausländer- und Integrationsgesetz (AIG), Asylgesetz (AsylG), Bürgerrechtsgesetz (BüG) sowie Freizügigkeitsabkommen (FZA) mit weiteren Erlassen, 5. Aufl., Zürich 2019.

7 Vgl. auch ASTRID EPINEY, «Brexit» und FZA, Zu den Perspektiven der Freizügigkeit zwischen der Schweiz und der EU im Gefolge des «Brexit», Jusletter vom 20. März 2017, N 4 ff.

8 Abkommen zwischen der Schweizerischen Eidgenossenschaft und dem Vereinigten Königreich von Grossbritannien und Nordirland über die Rechte der Bürgerinnen und Bürger infolge des Austritts des Vereinigten Königreichs aus der Europäischen Union und des Wegfalls des Freizügigkeitsabkommens (SR 0.142.113.672).

9 Abkommen vom 25. Februar 2019 zwischen der Schweizerischen Eidgenossenschaft und dem Vereinigten Königreich von Grossbritannien und Nordirland über die Rechte der Bürgerinnen und Bürger infolge des Austritts des Vereinigten Königreichs aus der Europäischen Union und des Wegfalls des Freizügigkeitsabkommens (AS 202186). 
soll sich auf den Inhalt der Teile eins (Grundbestimmungen) und zwei(Rechtebetreffend AnhangI) beschränken. ${ }^{10}$

5 Ziel des Abkommens ist es gemäss seinem Art.1, die unter dem FZA erworbenen Ansprüche zwischen der Schweiz und dem UK für die Zukunft zu sichern. So soll auch der Verpflichtung aus Art.23 FZA nachgekommen werden, auf welchen sich das Abkommen stützt. Art.23 FZA sieht bei einem Wegfall des FZA vor, dass die darauf gestützt erworbenen Rechte davon unberührt bleiben und die Anwartschaften in beiderseitigem Einvernehmen geschützt werden sollen. ${ }^{11}$ Im Interesse der Rechtssicherheit und der erleichterten Umsetzung werden diese erworbenen Ansprüche im Abkommen «ausgestaltet» und die Anwartschaften geregelt. ${ }^{12}$ Da es sich um den ersten Anwendungsfall des Art.23 FZA handelt, gab es noch keine Erfahrungen, auf die man hätte zurückgreifen können. Eine genauere Abgrenzung und Umschreibung der erworbenen Rechte einerseits und der Anwartschaften andererseits wurde in den Verhandlungen nicht thematisiert. ${ }^{13}$ Grundsätzlich kann davon ausgegangen werden, dass die erworbenen Rechte spezifische Rechtspositionen Einzelner umfassen, die durch bereits erfolgte Ausübung von Freizügigkeitsrechten entstanden sind, selbst wenn sie noch nicht geltend gemacht wurden, wie beispielsweise beim Familiennachzug. ${ }^{14}$

6 Der Begriff der Anwartschaften spielt vor allem im Versicherungsbereich eine Rolle und umschreibt zukünftige Rechte oder Leistungen, welche vom Eintritt gewisser Ereignisse abhängig sind. ${ }^{15}$ Für den in diesem Beitrag behandelten Teil der Freizügigkeitsrechte dürften vor allem die erworbenen Ansprüche von Bedeutung sein, weshalb die Anwartschaften an dieser Stelle nicht weiter ausgeführt werden. ${ }^{16}$

7 Das Abkommen ist mit dem FZA allgemein eng verbunden und enthält zahlreiche Verweise darauf. Sowohl Art.4 Abs. 5 als auch Art. 5 des Abkommens legen gewisse

10 Eine Diskussion der Teile drei (Koordinierung der sozialen Sicherheit), vier (Gegenseitige Anerkennung von Berufsqualifikationen) sowie fünf(Schlussbestimmungen) würde über den Rahmen dieses Beitrags hinausgehen.

11 Siehe hierzu Botschaft zum Abkommen (Fn. 1), S. 1035; diesbezüglich hatte die Schweiz eine klarere Ausgangslage als die EU, denn der Austritt gemäss Art.50 EUV sieht einen solchen Schutz erworbener Rechtenicht explizit vor, vgl. dazu ASTRID EPINEY, Brexit und die Schweiz: «Mind the Gap», SRIEL 2019, S. 249.

12 Botschaft zum Abkommen (Fn.1), S. 1035 und 1041.

13 Botschaft zum Abkommen (Fn.1), S. 1041.

14 EPINEY, «Mind the Gap» (Fn.11), S. 247.

15 EPINEY, «Mind the Gap» (Fn. 11), S. 248.

16 Für eine umfassende Abgrenzung siehe CHRISTA TOBLER, Und wenn das Abkommen wegfällt? Erworbene Rechte nach Art.23 FZA, in: Achermann et al. (Hrsg.), Jahrbuch für Migrationsrecht 2015/2016, Bern 2016; SARAH PROGIN-THEUERKAUF, Kündigung des Freizügigkeitsabkommens - Und dann?, «NCCR on the move»-Blog vom 28.Juni 2020; EPINEY, «Brexit» und FZA (Fn. 7), N10 ff.
Grundsätze für das Verhältnis des Abkommens zum FZA fest. Art. 5 Abs. 1 formuliert die allgemeine Regel, dass Verweise auf das FZA sich auf die Version das FZA beziehen, die unmittelbar vor dem 1. Januar 2021 anwendbar war. Art. 5 Abs. 2 stellt die gleiche Regel für Bezugnahmen auf Unionsrecht und unionsrechtliche Bestimmungen fest. Neben diesen Regelungen zur materiellen Parallelität enthält das Abkommen auch gewisse Vorschriften zur parallelen Auslegung und Anwendung der beiden Rechtsquellen.

Gemäss Art. 4 Abs. 5 des Abkommens sind die Bestim- 8 mungen, die sich auf das FZA und darin enthaltene unionsrechtliche Begriffe beziehen, in Übereinstimmung mit Art.16 Abs.2 FZA auszulegen. Letzterer statuiert für die Anwendung des FZA eine Berücksichtigungspflicht der Parteien jener EuGH-Rechtsprechung zu unionsrechtlichen Begriffen, die vor dem Zeitpunkt der Unterzeichnung ergangen ist. Zusätzlich werden gemäss «inzwischen ständiger ${ }^{\mathbf{1 7}}$ Rechtsprechung des Bundesgerichts grundsätzlich auch spätere Urteile des EuGH zu abkommensrelevanten unionsrechtlichen Bestimmungen beachtet, es sei denn, «triftige Gründe» sprächen dagegen. ${ }^{18}$ Dies ist insbesondere daraus abzuleiten, dass das FZA zum Ziel hat, im Bereich der Personenfreizügigkeit mit gewissen Ausnahmen eine möglichst parallele Rechtslage zwischen der Schweiz und der EU und ihren Mitgliedstaaten zu schaffen. ${ }^{19}$

Somit soll gemäss Art. 4 Abs. 5 des Abkommens für die 9 Auslegung des Abkommens jedenfalls die einschlägige Rechtsprechung des EuGH bis zum Datum der Unterzeichnung des FZA berücksichtigt werden. Weiter kann wohl davon ausgegangen werden, dass bereits ergangene Rechtsprechung von Schweizer Gerichten zum FZA in Auslegung des Art.16 Abs.2 FZA, welche eine Übernahme späterer EuGH-Urteile vorsieht, ebenfalls zu beachten sein wird. Unklar erscheint, ob bei der Auslegung des Art. 4 Abs. 5 des Abkommens analog zur Auslegung des Art.16 Abs.2 auch zukünftige relevante Rechtsprechung des EuGH berücksichtigt werden wird. Der Bundesrat hält diesbezüglich fest, Art. 4 Abs. 5 des Abkommens diene dazu, dass der statische Charakter des FZA auch im Abkommen zum Tragen komme. ${ }^{20}$ Der statische

17 BGE146II 89 E.4.3; vgl. jedoch eine neuere Rechtsprechung der strafrechtlichen Abteilung, welche dem FZA primär wirtschaftlichen Charakter zuspricht und bei der Einschränkung der Rechte nach Art. 5 Anhang I FZA keine enge, sondern eine dem Wortlaut entsprechende Auslegung erwägt, siehe insb. Urteil des Bundesgerichts 6B_378/2018 vom 22. Mai 2019 sowie EPINEY, Das Freizügigkeitsabkommen Schweiz - EU (Fn. 6), Fn. 34.

18 BGE136 II 5 E. 3.4; BGE139 II 393 E. 4.1.1; BGE140 II 112 E. 3.2; BGE142 II 35 E. 3.1; vgl. dazu EPINEY, Das Freizügigkeitsabkommen Schweiz-EU (Fn.6), N20 und dort Fn.33.

19 EPINEY, Das Freizügigkeitsabkommen Schweiz-EU (Fn. 6), N18.

20 Botschaft zum Abkommen (Fn.1), S. 1047. 
Charakter bedeutet, dass durch Verweis erwähntes EURecht (ob Rechtsakte oder Rechtsprechung) grundsätzlich in der Fassung gilt, die zum Zeitpunkt der Unterzeichnung massgeblich war. ${ }^{21}$

Das Abkommen hat in erster Linie zum Ziel, unter dem FZA erworbene Rechte zu sichern, bzw. sie gewissermassen in der Situation vor dem Austritt des UK aus der EU «einzufrieren». Insoweit wird daher keine parallele Rechtslage mit der EU mehr angestrebt und es scheint diesbezüglich nicht angezeigt, dass die zukünftigen Auslegungen des EuGH noch berücksichtigt werden müssen.

Weiter erwähnenswert sind verschiedene zeitliche Aspekte des Abkommens. Zum Zeitpunkt des Abschlusses des Abkommens war bereits klar, dass die EU und das UK eine Übergangsphase vereinbaren könnten, in welcher für eine gewisse Dauer im UK weiter EU-Recht (und damit das FZA) zur Anwendung kommen würde. Gleichzeitig bestand die Gefahr, dass es zu keiner solchen Einigung kommen und das UK vertragslos aus der EU «herausbrechen» könnte. Für die Schweiz und das UK galt es, jedenfalls ein Vakuum zu verhindern. Dementsprechend wurde in Art.2 lit.b des Abkommens der «festgelegte Stichtag» flexibel definiert. Je nachdem, wie sich diese Situation gestalten würde, sollte der festgelegte Stichtag entweder auf den Tag nach der Übergangsphase oder auf den Tag nach dem Verlust der EU-Mitgliedschaft durch das UK fallen. Dadurch, dass die EU und das UK letzlich eine Übergangsphase vereinbart haben, trat ersteres ein und der festgelegte Stichtag war somit der 1. Januar 2021. Diesem Datum kommt gleich mehrfach Bedeutung zu; es definiert den Moment, ab welchem die Parteien das Abkommen vorläufig anzuwenden hatten (Art. 36 Abs.3 lit.a des Abkommens). Ausserdem hat es insofern eine grosse Bedeutung, als dass es den Punkt darstellt, vor dem individuelle Rechte grundsätzlich erworben sein müssen, um vom Abkommen geschützt zu sein. ${ }^{22}$

21 Vgl. MATthias OESCH, Die bilateralen Abkommen Schweiz-EU und die Übernahme von EU-Recht, AJP 2017, S. 639.

22 Dies dürfte die in der Lehre teils aufgeworfene Frage beantworten, ob für das Erwerben von Rechten nach Art.23 FZA zeitlich der Tag des Aussprechens der Kündigung oder Tag der Wirksamkeit derselben als Stichtag entscheidend ist, vgl. ALVARO BORGHI (Fn. 6), S. 381; GAËTAN BLASER, in: Cesla/Nguyen (Hrsg.), Code annoté de droit des migrations, Vol. III: Accord sur la libre circulation des personnes (ALCP), Bern 2014, Art.23, S. 242f., N4; der Fall des Brexits ist zwar nicht gleich gelagert, da keine Kündigung ausgesprochen wurde, sondern das FZA für das UK aufgrund des Verlusts seiner EU-Mitgliedschaft wegfiel; trotzdem kann analog davon ausgegangen werden, dass auch bei einer allfälligen Kündigung des FZA der letzte Tag der Gültigkeit des Abkommens für die erworbenen Rechte entscheidend wäre. Dies erscheint überzeugend, da bis und mit diesem Tag das Abkommen ja vollumfänglich gilt, vgl. dahingehend auch TOBLER (Fn. 16), S. 45; PROGIN-THEUERKAUF (Fn.16).

\section{Aufenthaltsrechte}

Teil zwei des Abkommens übernimmt die Rechte betref- 12 fend Anhang I FZA, welcher die Freizügigkeit betrifft. Zunächst wird in diesem Teil der persönliche Geltungsbereich des Abkommens definiert.23 Er ist klar begrenzt, da er sich auf die Personen, welche bereits Rechte erworben bzw. Anwartschaften haben, beschränkt. Dies führt dazu, dass sowohl in der Schweiz als auch im UK neu zwei Kategorien von Staatsangehörigen des anderen Staates zu unterscheiden sind: Einerseits solche, die erworbene Rechte haben, und andererseits solche, die keine solchen Rechte haben. ${ }^{24}$ Auf letztere soll später näher eingegangen werden.

In erster Linie gilt das Abkommen für Schweizer Staats- 13 angehörige und Staatsangehörige des UK, welche ihr Recht auf Aufenthalt auf der Rechtsgrundlage des FZA vor dem 1. Januar $2021 \mathrm{im}$ jeweils anderen Staat ausgeübt haben und sich auch weiterhin dort aufhalten (Art.10 Abs. 1 lit.a und b des Abkommens, im Folgenden: hauptberechtigte Personen).

Auch die Familienangehörigen der hauptberechtigten 14 Personen werden vom Abkommen erfasst, wenn sie alternativ eine der folgenden Voraussetzungen erfüllen: i) sie hielten sich vor dem 1. Januar 2021 nach dem FZA im Aufnahmestaat auf und tun dies auch weiterhin; ii) sie sind mit Hauptberechtigten direkt verwandt und hielten sich vor dem 1. Januar 2021 ausserhalb des Aufnahmestaats auf, erfüllen aber die Bestimmungen von Art.3 Abs.2 lit.a-c Anhang I FZA («Familienangehörige» gemäss FZA) zum Zeitpunkt, in dem sie Aufenthalt nehmen möchten, um zur hauptberechtigten Person zu ziehen; iii) sie wurden, unabhängig davon, ob in oder ausserhalb des Aufnahmestaats, am oder nach dem 1.Januar 2021 als Kind einer hauptberechtigten Person geboren, und erfüllen die Bestimmungen von Art. 3 Abs. 2 lit.a-c Anhang I FZA zum Zeitpunkt, in dem sie Aufenthalt nehmen möchten, um zu einer hauptberechtigten Person zu ziehen; iv) sie werden innerhalb von fünfJahren nach dem festgelegten Stichtag der Ehegatte bzw. die Ehegattin einer hauptberechtigten Person, sofern sie vor dem Ende dieses Zeitraums Aufenthalt nehmen möchten, um zu dieser zu ziehen (Art. 10 Abs.1 lit.e Ziff.i-iv des Abkommens).

Bemerkenswert ist, dass in Ziff.iii für Kinder von haupt- 15 berechtigten Personen keine zeitliche Beschränkung festgehalten wird. Dies führt dazu, dass das Abkommen trotz

23 Dabei gilt dieser grundsätzlich für das gesamte Abkommen, wobe für die Teile drei und vier unberührt davon die Art.25, 26a und 29 jeweils den Geltungsbereich festlegen (Botschaft zum Abkommen [Fn.1], S. 1049).

24 Dabei dürfte es in der Anfangsphase in der Praxis Unterscheidungsschwierigkeiten geben. 
der beschränkten Anzahl berechtigter Personen ${ }^{25}$ voraussichtlich noch jahrzehntelang anwendbar sein wird, zumal die Rechte auf Lebenszeit garantiert sind (Art. 4 Abs.2). ${ }^{26}$

Eine zeitliche Begrenzung gilt hingegen für zukünftige Ehegatten hauptberechtigter Personen. Demgemäss können diese noch fünf Jahre lang, d.h. bis zum 31. Dezember 2025, «privilegiert» nach den Regeln des Abkommens nachgezogen werden; danach werden innerstaatliche Vorschriften anwendbar sein. ${ }^{27}$ Diesbezüglich enthält das Abkommen einen Vorteil im Vergleich zum Regime, das zwischen der EU und dem UK ausgehandelt wurde; im Austrittsabkommen ${ }^{28}$ fehlt eine entsprechende Regelung für zukünftige Ehegatten. ${ }^{29}$

17 Weiter ist zu beachten, dass der Familiennachzug auf Personen beschränkt ist, welche ein originäres Aufenthaltsrecht besitzen. Dies ergibt sich aus Art.15 des Abkommens: Grundsätzlich gibt es die Möglichkeit zum Wechsel von einem Aufenthaltsstatus zu einem anderen, z.B. vom Studenten zum Arbeitnehmer, wenn die Kriterien der entsprechenden Aufenthaltskategorie erfüllt sind. Jedoch können Familienangehörige mit von einer hauptberechtigten Person abgeleitetem Aufenthaltsrecht nicht zu einer hauptberechtigten Person gemäss Art.10 Abs.1 lit.a und b des Abkommens werden. Somit können sie auch keine Familiennachzugsrechte nach dem Abkommengeltend machen (vgl. Art.15 Satz2 des Abkommens). ${ }^{30}$ Art.12 garantiert, analog den einschlägigen Bestimmungen des FZA. Art.12 Abs.1 des Abkommens übernimmt alle im FZA vorgesehenen originären Aufenthaltsrechtskategorien: Aufenthalt mit Erwerbstätigkeit (Art. 2 Anhang I FZA), Unselbstständigerwerbstätige (Art. 6 Anhang I FZA), Selbstständigerwerbstätige (Art.12 Anhang I FZA), Personen mit eine Verbleiberecht (Art. 4 Anhang I FZA) sowie Personen ohne Erwerbstätigkeit (Art. 24 Anhang I FZA). ${ }^{31}$ Art.12 Abs.2 des Abkommens betrifft die Familienangehörigen und verweist ebenfalls direkt auf die einschlägigen FZA-Bestimmungen (Art. 3 und 4 Anhang I). Demgemäss sollen insbesondere die abgeleiteten

\footnotetext{
25 Aufgrund welcher auch auf eine Kündigungsklausel im Abkommen verzichtet wurden, vgl. Botschaft zum Abkommen (Fn. 1), S. 1046.

26 Unter dem Vorbehalt, dass die Schweiz und das UK etwas Gegenteiliges vereinbaren könnten.

27 Botschaft zum Abkommen (Fn.1), S. 1049.

28 Abkommen über den Austritt des Vereinigten Königreichs Grossbritannien und Nordirland aus der Europäischen Union und der Europäischen Atomgemeinschaft (ABl. C 384 I/01 vom 12. November 2019).

29 Zum Regime EU-UK: STEVE PEERS, The End - or a New Beginning? The EU/UK Withdrawal Agreement, Yearbook of European Law, Bd.39, Oxford 2020.

30 Vgl. Botschaft zum Abkommen (Fn.1), S. 1054.

31 Vgl. Botschaft zum Abkommen (Fn.1), S. 1050.
}

Aufenthaltsberechtigungen der Familienangehörigen ein Aufenthaltsrecht derselben Dauer verleihen wie das der Hauptberechtigten. ${ }^{32}$ Familienangehörige haben ausserdem weiterhin das Recht auf Zugang zu einer Erwerbstätigkeit bzw. auf Teilnahme am allgemeinen Unterricht sowie, im Falle von Kindern, an der Berufsbildung (vgl. auch Art.18 des Abkommens).

Bei diesen Aufenthaltsrechten haben die Behörden kei- 19 nen Ermessensspielraum zuungunsten der Betroffenen, wenn ein Rechtsanspruch gemäss FZA besteht (Art.12 Abs. 3 des Abkommens). Es dürfen auch keine weiteren Beschränkungen und Voraussetzungen für den Erwerb, Erhalt und Verlust von Aufenthaltsrechten eingeführt werden.

Bezüglich Ein- und Ausreiserechte für die vom Abkom- 20 men Berechtigten werden in Art.13 des Abkommens ebenfalls grundsätzlich die einschlägigen FZA-Regeln übernommen; benötigt wird grundsätzlich nur ein nationaler Personalausweis oder ein Reisepass ${ }^{33}$. Hierbei gibt es jedoch gewisse Besonderheiten; Art.13 Abs.1 Unterabsatz 2 hält dem UK die Möglichkeit offen, nach dem 31. Dezember 2025 keine Schweizer Identitätskarten mehr für die Einreise zu akzeptieren, sofern diese nicht gewissen Standards der biometrischen Identifizierung entsprechen. ${ }^{34}$ Weiter wird mit Art.13 Abs.3 darauf Rücksicht genommen, dass die Schweiz als Mitglied des Schengenraums möglicherweise spezielle Verpflichtungen haben könnte. Wenn die Schweiz aufgrund ihrer Schengen-Assoziierung in Zukunft Dokumente für die Ein- und Ausreise verlangt, die über die in Art.1 Anhang I FZA genannten hinausgehen - beispielsweise ein Visum - so kann das UK dies gegenüber der Schweiz ebenfalls tun.

Im Gegenteil zum FZA enthält das Abkommen neu Be- 21 stimmungen zum Daueraufenthaltsrecht. So können die Berechtigten nach nicht mehr als fünf Jahren ununterbrochenen $^{35}$ und rechtmässigen Aufenthalts ein solches verlangen (Art.14 Abs.1 des Abkommens). Dieses Daueraufenthaltsrecht entspricht in der Schweiz grundsätzlich der Niederlassungsbewilligung C, welche in Art.34 des Ausländer- und Integrationsgesetz ${ }^{36}$ geregelt ist. ${ }^{37}$

32 Botschaft zum Abkommen (Fn.1), S. 1050.

33 Sowie ein allfälliges Visum bei drittstaatsangehörigen Familienmitgliedern.

34 Schweizer Staatsangehörige, die nicht vom Abkommen erfasst sind, können schon ab 1. Oktober 2021 grundsätzlich nicht mehr mit der nicht-biometrischen Schweizer ID ins UK einreisen.

35 Damit der Aufenthalt als ununterbrochen gilt, darf der Berechtigte grundsätzlich nicht länger als sechs Monate vom Aufenthaltsstaat abwesend sein (Art.11 des Abkommens mit weiteren Verweisen auf das FZA).

36 Bundesgesetz über die Ausländerinnen und Ausländer und über die Integration vom 16. Dezember 2005 (Ausländer- und Integrationsgesetz, AIG; SR142.20).

37 Siehe auch Botschaft zum Abkommen (Fn. 1), S. 1053. 
Dafür braucht es prinzipiell einen Aufenthalt von zehn Jahren; gemäss behördlicher Praxis wird diese jedoch für gewisse Länder, darunter das UK, bereits nach fünf Jahren erteilt. ${ }^{38}$ Gewissermassen spiegelbildlich werden Schweizerinnen und Schweizer im UK diesbezüglich grundsätzlich gleichbehandelt wie EU/EWR-Bürgerinnen und -Bürger, das heisst gemäss der Richtlinie 2004/38/EG. Darin ist vorgesehen, dass nach fünfJahren ununterbrochenem rechtmässigem Aufenthalt ein Daueraufenthaltsrecht besteht (Art. 16 Richtlinie 2004/38/ EG). Diese Praxis wird in Art.14 des Abkommens aufgenommen und neu als klarer Rechtsanspruch festgehalten.

Dieses einmal erworbene Daueraufenthaltsrecht bleibt von Abwesenheiten vom Hoheitsgebiet des Aufnahmestaates von weniger als vier aufeinanderfolgenden Jahren unberührt (Art. 14 Abs. 2 des Abkommens). ${ }^{39}$ Jedoch kann der Aufnahmestaat verlangen, dass die Abwesenheit gemeldet werden muss, bzw. um die Aufrechterhaltung des Daueraufenthaltsrechts ersucht werden muss (Art.14 Abs.3 des Abkommens), wie dies in der Schweiz gemäss Art.61 Abs. 2 AIG der Fall ist. 40

23 Vor Erhalt des Daueraufenthaltsrechts wird das Aufenthaltsrecht nach dem Abkommen grundsätzlich bei einer unbegründeten Abwesenheit von mehr als sechs aufeinanderfolgenden Monaten hinfällig (Art.11 des Abkommens). ${ }^{41}$

Neu ist dem Abkommen im Vergleich zum FZA weiter dass der Aufnahmestaat verlangen kann, dass die vom Abkommen erfassten Personen einen neuen Aufenthaltsstatus beantragen, der ihnen erst die erwähnten Rechte verleiht (Art. 16 Abs.1 des Abkommens). Sowohl die Schweiz als auch das UK haben sich für ein solches System entschieden. ${ }^{42}$ Dies ist der Tatsache geschuldet, dass die auf dem Abkommen basierenden Aufenthaltsbewilligungen konstitutiven Charakter aufweisen. Hierin besteht ein signifikanter Bruch mit den Grundsätzen der Personenfreizügigkeit, was grosse Auswirkungen haben kann. Gerade im UK, wo Schweizerinnen und Schweizer lange keine Aufenthaltsbewilligung mehr beantragen mussten, könnten einige der Betroffenen die Frist für diese neue Bewilligung, die voraussichtlich am

\footnotetext{
38 MARC SPESCHA / PETER BOLZLI / FANNY DE WECK / VALERIO PRIULI, Handbuch zum Migrationsrecht, 4. Aufl., Zürich 2020, S. 169.

39 Hier ist das Verhältnis Schweiz-UK leicht nachteiliger als jenes EUUK: Gemäss Art.15 Abs.3 des Austrittsabkommens wird das Daueraufenthaltsrecht von EU-BürgerInnen im UK und BritInnen in der EU erst nach einer Abwesenheit von mehr als fünfJahren hinfällig.

40 Vgl. Rundschreiben des SEM, «Brexit Schutz der erworbenen FZA Rechte UK-Staatsangehöriger» vom 14. Dezember 2020, Ziff.2 Bst. I.

41 Botschaft zum Abkommen (Fn.1), S. 1050.

42 Botschaft zum Abkommen (Fn.1), S. 1054 f.; vgl. SEM-Rundschreiben «Brexit» (Fn. 40), Ziff. 2 Bst. A.
}

30.Juni 2021 abläuft ${ }^{43}$, verpassen und somit ihre Rechte verlieren. In der Schweiz ist diese Gefahr kleiner, da Britinnen und Briten bereits unter dem FZA eine Aufenthaltsbewilligung beantragen mussten. Diesbezüglich hält das Abkommen jedenfalls fest, dass für eine aus berechtigten Gründen verspätete Anmeldung eine Nachfrist zu gewähren ist (Art.16 Abs.1 lit.d des Abkommens).

Neu ist diesbezüglich ebenfalls, dass für das Ausstellen 25 der Aufenthaltsdokumente eine systematische Prüfung der Strafregister zulässig ist, welche einzig dazu dient, zu eruieren, ob Beschränkungen nach Art.17 des Abkommens anwendbar sind (Art. 16 Abs.1 lit.o des Abkommens).

Auch in Bezug auf die Beendigung des Aufenthaltsrechts 26 stellt das Abkommen neue Regeln auf. Für eine Beendigung des Aufenthaltsrechts aufgrund strafbaren Verhaltens der Berechtigten ist für Verhalten vor dem 1.Januar 2021 Art. 5 Anhang I FZA massgeblich, für späteres Verhalten hingegen das innerstaatliche Recht (Art. 17 des Abkommens).

Für Straftaten, die vor Wegfall des FZA begangen wurden, 27 ist gemäss Art. 4 Abs. 5 des Abkommens somit weiterhin Art.16 Abs.2 FZA einschlägig, d.h. die Rechtsprechung des EuGH ist zu berücksichtigen. Gemäss dieser vom Bundesgericht rezipierten Rechtsprechung muss somit persönliches Verhalten vorliegen, welches eine gegenwärtige und hinreichend schwere Gefährdung der öffentlichen Ordnung darstellt ${ }^{44}$; hierbei kommt es auf das Rückfallrisikoan, d.h. es wird eine hinreichende Wahrscheinlichkeit verlangt, dass die ausländische Person auch künftig die öffentliche Ordnung stören wird. ${ }^{45}$ Hingegen sind auf Straftaten nach dem 1.Januar 2021 in der Schweiz insbesondere Art. 62 und 63 des AIG anwendbar; so kann beispielsweise - unter Wahrung der Verhältnismässigkeit bereits eine Freiheitsstrafe von mehr als zwölf Monaten zum Widerruf der Aufenthaltsbewilligung führen. ${ }^{46}$

\section{Grenzgängerinnen und Grenzgänger}

Auch erworbene Rechte von Schweizer Staatsangehöri- 28 gen und Staatsangehörigen des UK, die vor dem 1.Januar 2021 ihre Rechte als (selbstständige oder abhängig beschäftigte) Grenzgängerinnen und Grenzgänger im jeweils anderen Land ausgeübt haben und diese auch

43 Vgl. Überblick der britischen Regierung, Apply to the EU Settlement Scheme (settled and pre-settled status).

44 Anstelle vieler: BGE129 II 215 E. 7.2 und 7.3; BGE130 II 176 E. 4.3.1 und E. 4.2 mit Verweis auf Urteil des EuGH 30/77 vom 27. Oktober 1977 (Bouchereau) sowie Urteil des EuGH C-348/96 vom 19. Januar 1999 (Calfa); vgl. NINA BURRI / VALERIO PRIULI, Landesverweisung und Freizügigkeitsabkommen, AJP 2017, S. 893.

45 BGE139 II121 E. 5.3; vgl. Botschaft zum Abkommen (Fn.1), S. 1057. 46 Botschaft zum Abkommen (Fn.1), S. 1057. 
weiter ausüben, werden vom Abkommen geschützt (Art.10 Abs.1 lit.c und d). So werden deren Rechte aufberufliche und geografische Mobilität und Gleichbehandlung nach den Art.8, 9, 14 sowie 15 Anhang I FZA gewahrt (Art. 20 Abs.1 des Abkommens). In diesem Zusammenhang können sie weiter ein- und ausreisen (Art. 20 Abs.2 des Abkommens). Die Beendigung des Aufenthalts richtet sich nach Art.17 des Abkommens (Art. 20 Abs.3, vgl. Ausführungen oben).

Grenzgängern kann verlangt werden, dass sie ein Dokument beantragen, welches - analog zur oben beschriebenen Aufenthaltsbewilligung - entweder bescheinigt, dass sie über die genannten Rechte verfügen, oder welches diese Rechte gar erst verleiht (Art. 21 Abs.1 lit.a und b des Abkommens).

Dabei erfasst der verwendete Begriff des Grenzgängers nur britische und schweizerische Staatsangehörige, die im einen Staat arbeiten und im jeweils anderen arbeiten (Art. 9 lit.b des Abkommens). Staatsangehörige der EU, welche in der Schweiz oder im UK leben und im jeweils anderen Staat arbeiten, werden vom Abkommen nicht erfasst. Auf diese Unstimmigkeit wird weiter unten eingegangen (II.5.).

\section{Dienstleistungserbringerinnen und -erbringer}

31 Schliesslich sind auch Dienstleistungserbringer vom Abkommen geschützt, wenn auch in sehr begrenztem Umfang. Diese sind in Bezug auf Art. 5 FZA sowie Art.17 Anhang I FZA berechtigt, am oder nach dem 1.Januar 2021 im Hoheitsgebiet der anderen Partei Dienstleistungen zu erbringen, deren Dauer 90 tatsächliche Arbeitstage pro Kalenderjahr nicht überschreitet. Dafür werden zwei Bedingungen gestellt: Erstens muss die Erbringung der Dienstleistung auf einem schriftlichen Vertrag (inkl. elektronische Form) basieren, der bereits vor dem 1. Januar 2021 abgeschlossen wurde (Art. 23 Abs.1 Ziff.i des Abkommens). Zweitens wird eine zeitliche Maximaldauer der Dienstleistungserbringungen festgelegt; diese dürfen bis höchstens zum 31. Dezember 2025 erbracht werden (Art. 23 Abs.1Ziff.ii des Abkommens). Diese zeitliche Begrenzung sowie die Notwendigkeit eines schriftlichen Vertrags bedeuten eine Abkehr von den noch unter dem FZA geltenden Regeln. Mit dem Erfordernis eines bereits bestehenden schriftlichen Vertrages wird immerhin die Rechtssicherheit gewährleistet und das in den Vertrag bestehende Vertrauen geschützt.

Der Begriff der Dienstleistungserbringer gilt ausschliesslich für schweizerische und britische Staatsangehörige, die sich im Gebiet der Schweiz oder des UK niedergelassen haben und im jeweils anderen Staat eine Dienstleis- tung erbringen (Art. 9 lit.d des Abkommens). Nicht erfasst sind somit wieder EU-Staatsangehörige, die sich in der Schweiz oder dem UK niedergelassen haben und im jeweils anderen Staat eine Dienstleistung erbringen. Ausserdem können sich Schweizer Staatsangehörige, die in einem EU-Staat niedergelassen sind und als selbstständige Dienstleistungserbringer im UK tätig werden wollen, nicht auf das Abkommen berufen. Gleiches gilt für britische Staatsangehörige, die in einem EU-Staat niedergelassen sind. ${ }^{47} \mathrm{Zu}$ denken ist hier beispielsweise an selbstständige britische Taxifahrer und-fahrerinnen, welche in Frankreich ihre Niederlassung haben, aber vor allem Gäste vom Flughafen Genf aus befördern.

Hierbei sowie amähnlich gelagerten Beispiel des Begriffs 33 der Grenzgängerinnen und Grenzgänger zeigt sich die fehlende Triangulierung des Abkommens mit der EU. Auf diese Fälle soll sogleich zurückgekommen werden.

\section{Verhältnis zu Art.23 FZA}

Wie dargelegt verweist das Abkommen insbesondere bei 34 sechs Punkten auf innerstaatliche Rechtsvorschriften, nicht auf das FZA: (1) Nachzug des Ehegatten nach dem Stichtag; (2) Daueraufenthaltsstatus; (3) Anspruch auf Nachzug von Familienangehörigen; (4) deklaratorischer Charakter der Aufenthaltsbewilligung; (5) öffentliche Ordnung (systematische Überprüfung des Strafregisters und Beschränkung des Aufenthaltsrechts nach innerstaatlichem Recht); und (6) Dienstleistungserbringung (zeitliche Begrenzung und Erfordernis eines schriftlichen Vertrags). ${ }^{48}$ Auch darüber hinaus gibt es nicht immer eine vollständige Übereinstimmung mit dem FZA, wie das Beispiel der Definition der Grenzgänger zeigt. Diesbezüglich liegt die Frage nahe, welches Verhältnis diese Regelungen im Abkommen zu jenen im FZA haben. Grundsätzlich besteht die Gefahr, dass (Migrations-)Behörden die Position vertreten, dass alle erworbenen Rechte im Abkommen abschliessend geregelt werden und daneben keine weiteren erworbenen Rechte bestehen. Diese Grundannahme wäre jedoch mit dem Sinn und Zweck des Art.23 FZA nicht vereinbar.

Wie oben ebenso dargelegt umfasst der Begriff des Grenz- 35 gängers nur schweizerische und britische Staatsangehörige, nicht aber solche der EU, welche in der Schweiz oder im UK leben und im jeweils anderen Staat arbeiten. Der Bundesrat hält in seiner Botschaft zum Abkommen fest, dass sich diese Grenzgänger mit EU/EFTA-Staatsangehörigkeit nach Wegfall des FZA mit dem UK weder auf das Abkommen noch auf das FZA berufen könnten,

47 Botschaft zum Abkommen (Fn.1), S. 1049.

48 Vgl. Botschaft zum Abkommen (Fn.1), S. 1043. 
um Grenzgängerstatus zu erlangen. ${ }^{49}$ Hingegen hält das Staatssekretariat für Migration (SEM) in seinem Rundschreiben von Dezember 2020 im Vorfeld des Inkrafttretens des Abkommens an die zuständigen Migrationsbehörden fest, dass diese Grenzgängerinnen und Grenzgänger direkt von Art.23 FZA geschützt seien; so sei ihnen nach Ablauf des Ausweises direkt gestützt darauf weiterhin eine Grenzgängerbewilligung zu erteilen. ${ }^{50}$ Gleiches gelte für selbstständige Dienstleistungserbringer mit EU/EFTA-Staatsangehörigkeit, die ihren Wohnsitz im UK haben. Ihre Rechte seien gestützt auf Art.23 FZA zu schützen, wenn sie analog die Bedingungen für Dienstleistungserbringer aus dem neuen Abkommen erfüllen. ${ }^{51}$ der Schutz dieser erworbenen Rechte mit der neuen restriktiveren Regelung im Abkommen ausgeschlossen, besagt das SEM-Rundschreiben klar das Gegenteil. Nach der hier vertretenen Ansicht ist letztere Auslegung vorzugswürdig. Das Abkommen macht klar, dass die Rechte der Grenzgängerinnen und Grenzgänger ziemlich umfassend zu den geschützten erworbenen Rechten zählen; der Standpunkt, dass nur gewisse Staatsangehörige diese Rechte erworben hätten, andere hingegen nicht, ist kaum nachzuvollziehen und würde in Bezug auf den Grundsatz der Nichtdiskriminierung (vgl. Art. 2 FZA), welcher in dieser Beziehung indirekt durch Art.23 FZA noch Wirkung entfalten dürfte, Fragen aufwerfen. So sollte grundsätzlich die Möglichkeit bestehen, dass ein Tatbestand, der vom Abkommen nicht erfasst ist, direkt von Art.23 FZA geregelt bzw. geschützt ist.

37 Anders verhält es sich in Fällen, in denen das neue Abkommen klar etwas entgegen dem FZA regelt, also wo die neue Regelung die alte ausschliesst, wie beim Beispiel des konstitutiven Charakters der Aufenthaltsbewilligung. Freilich steht es der Schweiz und dem UK offen, mit einem völkerrechtlichen Vertrag ihre Beziehung neu und in Abweichung vom FZA zu ordnen, trotz Art.23 FZA. Denn eine «Ewigkeitsgarantie» stellt dieser nicht dar; vielmehr kann jederzeit etwas anderes vereinbart werden. ${ }^{52}$ Gerade in diesem Beispiel sind jedoch die potenziellen Auswirkungen auf die Betroffenen gross. Das SEM ist diesbezüglich der Ansicht, dass UK-Staatsangehörige, die sich verspätet (d.h. nach dem 31. Dezember 2020) anmelden, ebenfalls unter das neue Abkommen fallen, wenn sie belegen können, dass sie vor dem 1.Januar 2021 von

\footnotetext{
49 Botschaft zum Abkommen (Fn.1), S. 1048.

50 SEM-Rundschreiben «Brexit» (Fn. 40), Ziff.2 Bst. C (iii).

51 SEM-Rundschreiben «Brexit» (Fn. 40), Ziff.3 und dort Fn.11.

52 PROGIN-THEUERKAUF (Fn.16); EPINEY, «Mind the Gap» (Fn.11), S. $247 \mathrm{f}$.
}

ihren Freizügigkeitsrechten Gebrauch gemacht haben. ${ }^{53}$ Das UK hingegen äussert die Rechtsansicht, dass Personen, welche unter das Abkommen fallen, sich aber zu spät oder nicht anmelden, als sich illegal im Land aufhaltend gelten werden. ${ }^{54}$ Diese Kategorisierung kann rückgängig gemacht werden, wenn bei einer verspäteten Anmeldung «reasonable grounds», das heisst gute Gründe, geltend gemacht werden können, weshalb keine frühere Anmeldung möglich war.

Auch in anderen Bereichen, in denen das Abkommen re- 38 striktiver ist als noch das FZA, wird für den Einzelfall zu eruieren sein, ob die neue Regelung die alte ausschliesst oder ob das Abkommen sich über einen gewissen Sachverhalt lediglich ausschweigt und somit Platz lässt für einen subsidiären Schutz durch Art.23 FZA. Es dürfte sich in jedem Fall schwieriger gestalten, im neuen Abkommen nicht explizit geregelte Rechte rechtsgültig geltend zu machen. Es bleibt abzuwarten, ob und inwiefern es zu einer richterlichen Klärung kommen wird.

\section{Neue Beziehung im Migrationsbereich}

Damit verbleibt die Frage, wie das Verhältnis zwischen 39 der Schweiz und dem UK sich neu in jenen Rechtsbereichen gestaltet, welche bislang durch das FZA geregelt waren.

Bereits im Februar 2020 wurden exploratorische Gesprä- 40 che zwischen der Schweiz und dem UK aufgenommen, um Möglichkeiten der zukünftigen Migrationszusammenarbeit zu erforschen. ${ }^{55}$ Auf ein umfassendes Abkommen hat man sich in diesem Bereich nicht geeinigt, wohl auch zumal die Stimmung und der politische Wille in beiden Ländern keine weiter erleichterte Migration anzeigen. Seitens der Schweiz setzt Art.121a der Bundesverfassung 56 hierbei gewisse Grenzen. So dürften die eigenständige Zuwanderungssteuerung der Schweiz (Art. $121 \mathrm{a}$ Abs.1) sowie die Begrenzung der Zulassung (Art.121a Abs.2 und 3) durch einen neuen völkerrechtlichen Vertrag mit dem UK nicht verunmöglicht bzw. missachtet werden (Art.121a Abs.4).57 Diese Verfassungsbestimmung

53 SEM-Rundschreiben «Brexit» (Fn. 40), Ziff.1 Bst. B.

54 Brief der Home Secretary Priti Patel an das Home Affairs Committee des House of Commons, 14. April 2020, Nr.12.

55 Medienmitteilung des Bundesrats vom 18. Dezember 2020 (Schweiz und UK verstärken bilaterale Migrationszusammenarbeit mit einem Memorandum of Understanding).

56 Bundesverfassung der Schweizerischen Eidgenossenschaft vom 18. April 1999 (BV; SR101).

57 PETER UEBERSAX, in: Belser/Waldmann/Epiney (Hrsg.), Basler Kommentar zur Bundesverfassung, Basel 2015, Art.121a BV N68ff. 
stellte für das Abkommen über die erworbenen Rechte kein Hindernis dar, da es vorrangig Rechte von Personen regelt, die sich schon in der Schweiz befinden bzw. weil es keine neuen völkerrechtlichen Verpflichtungen eingeht, welche nicht bereits unter dem FZA bestanden haben. ${ }^{58}$

41 Immerhin wurde am 18. Dezember 2020 ein Memorandum of Understanding (MoU) unterzeichnet. Diese rechtsunverbindliche Absichtserklärung soll als Grundlage für die zukünftige Zusammenarbeit mit Hilfe von Expertengruppen, Informationsaustausch und Capacity Building dienen - vor allem bei den geteilten prioritären Schwerpunkten gegenseitige Zugang zum Arbeitsmarkt, «Mobilität» sowie Koordination im Asylbereich. ${ }^{59}$

42 Darüber hinaus wurde für die Dienstleistungserbringung am 14. Dezember 2020, kurz vor Ende der Übergangsphase, das Abkommen über die Mobilität von Dienstleistungserbringern ${ }^{60}$ abgeschlossen. Dieses ist vorerst auf zwei Jahre befristet und sieht unterschiedliche Regime für die beiden Parteien vor (vgl. Art.12): Der Zugang für britische Dienstleistungserbringer in der Schweiz wird ohne Bedarf einer Aufenthaltsbewilligung wie bisher für bis zu 90 Arbeitstage pro Kalenderjahr unter Einbezug des bereits bekannten Meldeverfahrens weitergeführt (siehe insbesondere Art.2, 3 und 4 Anhang I). Im UK wird schweizerischen Dienstleistungserbringern der Zugang zu gewissen höher qualifizierten Dienstleistungssektoren gewährleistet, welche in Ziff.9 des Anhangs II abschliessend aufgelistet sind. Dabei werden diesen Dienstleistungserbringern gewisse Vorzüge zuteil: Einerseits müssen sie keine Englischkenntnisse nachweisen und andererseits unterliegen sie nicht der wirtschaftlichen Bedarfsprüfung im UK. ${ }^{61}$ Sie erhalten den Zugang zum UK-Markt mit einem sogenannten «Tier 5 International Agreement»-Visum ${ }^{62}$ für 12 Monate in einem Zeitraum von 24 Monaten (Ziff. 11 Anhang II).

43 Im Übrigen kommen im Bereich Migration innerstaatliche Vorschriften zur Anwendung. In der Schweiz gelten Britinnen und Briten neu als Drittstaatsangehörige und fallen somit unter den Anwendungsbereich des AIG, inklusive Kontingentierung der Aufenthaltsbewilligungen und Inländervorrang. Um die Auswirkungen dieser Veränderung abzufedern und die Flexibilität für die Wirtschaft

58 Botschaft zum Abkommen (Fn. 1), S. 1076.

59 Medienmitteilung des Bundesrats vom 18. Dezember 2020 (Fn. 55).

60 Befristetes Abkommen zwischen der Schweizerischen Eidgenossenschaft und dem Vereinigten Königreich von Grossbritannien und Nordirland über die Mobilität von Dienstleistungserbringern (SR 0.946.293.671.2).

61 Medienmitteilung des Bundesrats vom 4. Dezember 2020 (Schweiz UK: Bundesrat genehmigt Abkommen zur Mobilität von Dienstleistungserbringern).

62 UK Government, UK-Switzerland Services Mobility Agreement explainer, 4. Dezember 2020. zu gewährleisten, hat der Bundesrat für das Jahr 2021 vorerst ein Sonderkontingent für Aufenthaltsbewillgungen für Britinnen und Briten beschlossen. ${ }^{63}$ Dieses beträgt insgesamt 3'500 Bewilligungen: 1'400 Kurzaufenthaltsbewilligungen L sowie 2'100 Aufenthaltsbewilligungen B (Art.19b und Art.20bi.V.m. Ziff.7 Anhang 1 und Ziff.7 Anhang 2 VZAE64). Diese separaten UK-Kontingente können 2022 in das Drittstaatenkontingent miteinbezogen werden, sollte es bis dann zu keiner Einigung über ein allfälliges zukünftiges Migrationsregime gekommen sein. ${ }^{65}$

Auf der anderen Seite gilt für Schweizerinnen und Schwei- 44 zer, die ins UK übersiedeln wollen, neu das sogenannte Points-Based Immigration System ${ }^{66}$ Dieses gilt seit dem 1.Januar 2021 grundsätzlich für alle Einwanderer ins UK und inspiriert sich am australischen Immigrationssystem. Dabei werden Punkte verteilt für gewisse Attribute, beispielsweise Englischkenntnisse, ein konkretes Jobangebot oder ein Doktorat in relevanten Bereichen. Erst mit 70 erreichten Punkten kann die im Vorfeld zu beantragende Bewilligung erteilt werden. ${ }^{67}$

\section{Schluss}

Das Abkommen über die erworbenen Rechte der Bürge- 45 rinnen und Bürger hat viel zur Rechtssicherheit für die Personen beigetragen, die bislang unter das FZA fielen. Im Bereich, der in diesem Beitrag behandelt wurde, widerspiegelt es grossteils die Rechte, wie sie unter dem FZA gegolten haben. Dennoch gestaltet sich die Situation in einzelnen Bereichen anders, und dort meist restriktiver als noch unter dem FZA, wie beispielsweise beim neu konstitutiven Charakter der Aufenthaltsbewilligung. Die Anwendung des Abkommens als erste Praxisausprägung des Art.23 FZA wird in naher Zukunft zweifellos weitere auslegungsbezogene Fragestellungen aufzeigen.

Für Personen, die ab 2021 zwischen der Schweiz und dem 46 UK migrieren wollen, gilt ein neues Regime. Abgesehen vom befristeten Abkommen zur Dienstleistungserbringung wurde zwischen der Schweiz und dem UK kein neues umfassendes Migrationsabkommen gefunden. Im Übrigen gelten die nationalen Vorschriften, welche in der Schweiz in einer Kontingentierung, im UK in einem Punkte-basierten Bewilligungssystem bestehen.

63 Medienmitteilung des Bundesrats vom 18. Dezember 2020 (Fn. 55). 64 Verordnung über Zulassung, Aufenthalt und Erwerbstätigkeit vom 24. Oktober 2007 (VZAE; SR142.201).

65 Medienmitteilung des SEM vom 25. November 2020 (Bundesrat legt Kontingente 2021 für Erwerbstätige aus Drittstaaten und dem Vereinigten Königreich fest).

66 Medienmitteilung des UKHome Office vom 1. Dezember 2020 (Pointsbased immigration system opens).

67 Siehe UK Government, New immigration system: what you need to know, 16. May 2021. 
47 Insgesamt sind die Möglichkeiten der Migration zwischen den beiden Ländern sehr viel restriktiver geworden, was vor dem politischen Hintergrund nicht überrascht. Es bleibt abzusehen, was auf das MoU vom Dezember 2020 in der Beziehung zwischen der Schweiz und dem UK noch folgen mag. 